\title{
Correction to: Response inhibition deficits are positively associated with trait rumination, but attentional inhibition deficits are not: aggressive behaviors and interpersonal stressors as mediators
}

\author{
Akira Hasegawa $^{1}$ (D) Noboru Matsumoto $^{2}$ (D) Yuko Yamashita $^{3} \cdot$ Keisuke Tanaka $^{4}$ (D) Jun Kawaguchi ${ }^{5,6}$ (D) . \\ Tetsuya Yamamoto ${ }^{7}$ iD
}

Published online: 1 November 2021

○) Springer-Verlag GmbH Germany, part of Springer Nature 2021

\section{Correction to: Psychological Research https://doi.org/10.1007/s00426-021-01537-y}

In the original publication of the article, the information listed in Table 2 was not formatted correctly (1) all variables (e.g., "Depression") are written in one or two lines, and (2)
95\% confidence intervals are not broken (i.e., written in one line; e.g., [0.663, 0.790]). Hence this has been corrected and published in the erratum article as given below:
The original article can be found online at https://doi.org/10.1007/ s00426-021-01537-y.

Akira Hasegawa

mail-ad.of.hasse@tokaigakuin-u.ac.jp

Noboru Matsumoto

noborum@shinshu-u.ac.jp

Yuko Yamashita

yamashitayk10@gmail.com

Keisuke Tanaka

keisuke@juen.ac.jp

Jun Kawaguchi

jun.kawaguchi.cogpsy@gmail.com

Tetsuya Yamamoto

t.yamamoto@tokushima-u.ac.jp

1 Faculty of Human Relations, Tokai Gakuin University, 5-68

Naka-kirino, Kakamigahara City, Gifu 504-8511, Japan
2 Division of Psychology, Faculty of Arts, Shinshu University, Asahi 3-1-1, Matsumoto, Nagano 390-8621, Japan

3 Mutsumi Hospital, 3-11-23, Minamiyaso-cho, Tokushima 770-0005, Japan

4 Graduate School of Education, Joetsu University of Education, 1-Yamayashiki-machi, Joetsu-shi, Niigata 943-8512, Japan

5 Department of Psychology, Otemon Gakuin University, 2-1-15, Nishiai, Ibaraki City, Osaka 567-8502, Japan

6 Graduate School of Informatics, Nagoya University, Furo-cho, Chikusa-ku, Nagoya, Aichi 464-8601, Japan

7 Graduate School of Technology, Industrial and Social Sciences, Tokushima University, 1-1, Minamijosanjima-cho, Tokushima 770-8502, Japan 


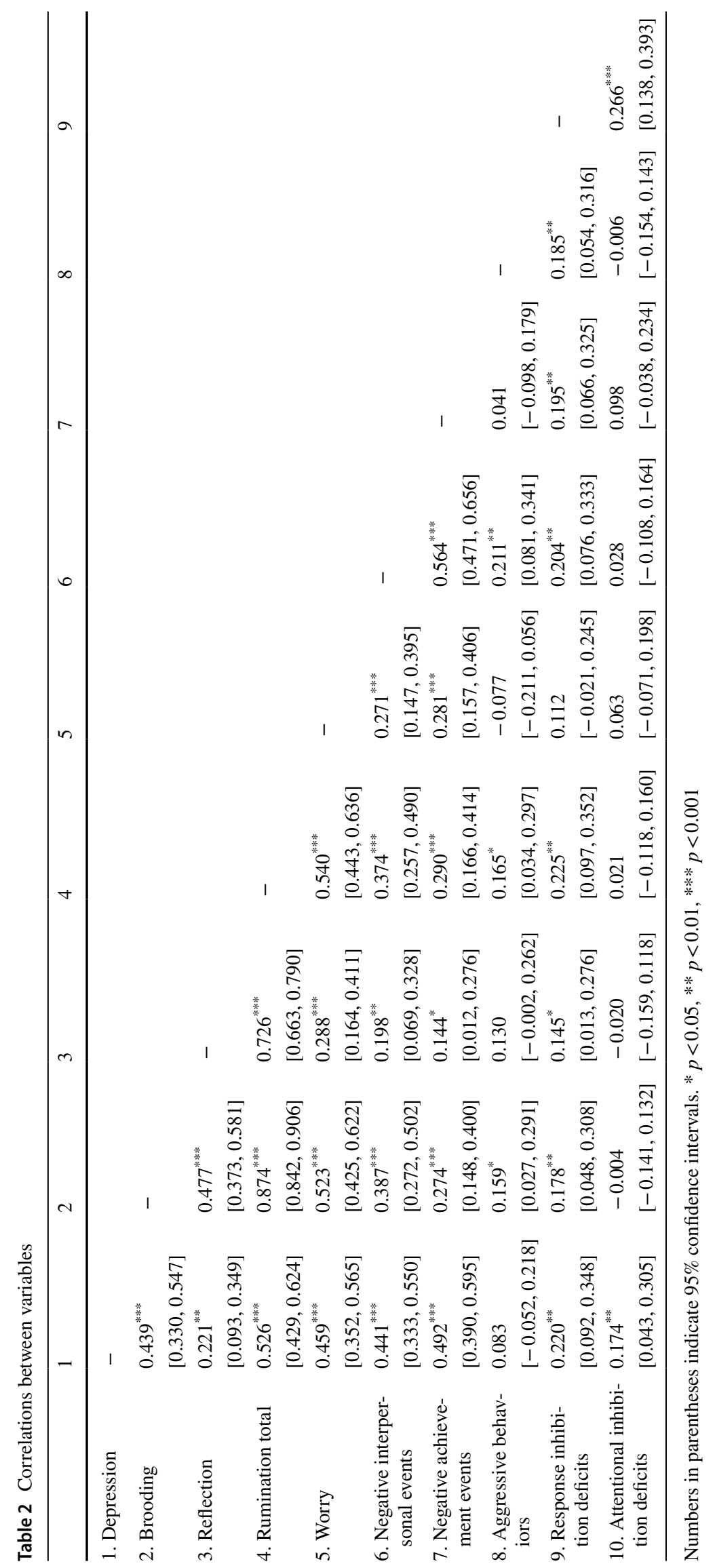


Publisher's Note Springer Nature remains neutral with regard to jurisdictional claims in published maps and institutional affiliations. 